\title{
GELES Y MEMBRANAS
}

\author{
X. Gascó \\ Unidad de Pie y Tobillo. Hospital Son Llàtzer. \\ Pieytobillo.es. (Clínica Rotger - Quirónsalud). Palma de Mallorca
}

\section{Introducción}

Las lesiones osteocondrales de astrágalo (LOA) son frecuentes y se diagnostican principalmente en jóvenes entre la segunda y la cuarta décadas, muchas veces en relación con traumatismos o actividad deportiva ${ }^{(1)}$. Hasta un $50 \%$ de los esguinces o fracturas de tobillo pueden ser causantes de algún tipo de lesión osteocondral ${ }^{(2)}$. La clínica dolorosa es en ocasiones limitante y, sin tratamiento, pueden evolucionar a degeneración articular progresiva que ha sido relacionada con el aumento local permanente de citocinas $^{(3,4)}$. Además, el cartílago articular es un tejido anervado, avascular e hipocelular, nutrido únicamente por el líquido sinovial, por lo que tiene muy poca capacidad de reparación endógena.

Estudios previos han demostrado que en lesiones sintomáticas de grado III o IV en adultos, el tratamiento quirúrgico obtiene mejores resultados ${ }^{(1,2,4-7)}$. Para ello existen diversos métodos de tratamiento. Ninguno de ellos (estimulación de médula ósea subcondral, terapias basadas en cultivos celulares de condrocitos o auto/ aloinjertos osteocondrales) ha sido suficientemente estudiado hasta ahora en términos de evidencia ${ }^{(7-11)}$, comunicándose resultados algo dispares. Posiblemente por este motivo, siguen utilizándose sin una clara indicación ni algoritmo terapéutico.

El objetivo de cualquiera de estas técnicas es lograr una reparación estructural de la lesión, lo más similar posible al cartílago nativo ${ }^{(2,5,6)}$, asegurando así su durabilidad para una buena función articular, no dolorosa a largo plazo. Obviamente, el método ideal sería el que lograra estos objetivos de la forma más sencilla y reproducible posible, en un único tiempo quirúrgico, minimizando las complicaciones, disminuyendo el tiempo de recuperación postoperatorio y con el menor coste económico.

La estimulación de médula ósea (EMO) artroscópica (perforaciones, micro- o nanofracturas) sigue considerándose el tratamiento de elección inicial para LOA me- 
nores de $15 \mathrm{~mm}^{(2,5,6,12,13)}$, ya que cumple casi todos los objetivos anteriores. Sin embargo, algunos estudios demuestran que la cantidad y calidad del fibrocartílago obtenido no posee las mismas características biológicas y biomecánicas que el cartílago hialino, lo que explicaría el deterioro de los resultados clínicos a medio-largo plazo.

Alternativas para lesiones más grandes o con importantes defectos o quistes subcondrales son los trasplantes o injertos osteocondrales $(2,5,13,14)$, que en estos casos han demostrado resultados más duraderos, pero con las limitaciones de potencial morbilidad en la zona donante (habitualmente rodilla homolateral) y que suelen requerir artrotomías u osteotomías del tobillo para su realización. En cuanto al uso de aloinjertos, también debido a sus potenciales complicaciones y resultados publicados, la evidencia existente sugiere que sea reservado para lesiones quísticas muy grandes o técnicas de rescate ${ }^{(2,5,13,15)}$.

El cultivo e implante de condrocitos autólogos $(\mathrm{ACl})$ también ha sido motivo de numerosos trabajos en los últimos años, simplificando su técnica asociando el uso de membranas de colágeno $(\mathrm{MACl})^{(2,5,12,13)}$. A pesar de que parece que el cartílago obtenido es muy similar al hialino, sus limitaciones siguen siendo un alto coste económico, el requerir de dos tiempos quirúrgicos, la preexistencia de un buen sustrato óseo subcondral ${ }^{(13,15,16)}$ y unas instalaciones que pocos centros tienen disponibles. Además, algunos estudios han concluido en similares resultados clínicos que las técnicas de EMO a medio plazo(2,5,12).

Por otro lado, en la última década, grupos de investigación relacionados con la bioingeniería y la biología molecular han promocionado la utilidad de coadyuvantes biológicos como el ácido hialurónico ${ }^{(17)}$, el plasma rico en plaquetas (PRP) $)^{(2,4,12)}$, el aspirado de médula ósea con concentración de células mesenquimales pluripotenciales ${ }^{(15,16)}$, el implante de cartílago juvenil particulado o el uso de "andamiajes" mediante geles o membranas de colágeno, para implementar los procesos reparativos naturales, favoreciendo la proliferación y diferenciación celular, garantizando así mejores y más duraderos resultados sin aumentar la complejidad o potenciales complicaciones.

Estos métodos, que suponen un aumento del gasto sanitario no desdeñable, se han difundido ampliamente por nuestro país en los últimos años, muchas veces más potenciados por la in- dustria que por la evidencia científica. Sin embargo, van apareciendo publicaciones con series más amplias y seguimientos más largos, y unos resultados que parecen ser prometedores.

\section{Objetivos}

El objetivo de este trabajo es revisar la bibliografía reciente y relevante respecto a la utilidad de geles y membranas de colágeno para el tratamiento de las LOA. Más concretamente, presentar la evidencia actualmente disponible que justifique su utilización y analizar por separado los diferentes productos actualmente disponibles en el mercado, con sus indicaciones, técnicas de implantación y resultados publicados.

En este capítulo nos limitaremos únicamente a su aplicación aislada o asociada a técnicas de EMO. Su utilización en el trasplante de condrocitos de segunda generación ( $\mathrm{MACl}$ ) es revisado en la otra sección de este capítulo de la monografía.

\section{Material y métodos}

Se ha realizado una búsqueda en MEDLINE (mediante el motor de búsqueda PubMed) de trabajos/artículos publicados entre 2001 y el año actual.

Como parámetros de búsqueda se han combinado los términos: osteochondral, lesions, talus, bone marrow stimulation, collagen scaffolds (collagen*). Utilizando todos ellos se obtuvo un solo artículo. Cambiando el parámetro membrane por gel no se obtuvo ningún resultado.

Ampliando los parámetros de búsqueda a unos términos más generales, se obtuvieron 45 artículos, de los cuales 21 han sido publicados en el último año y 38 (84\%) en los últimos 5 años.

Se han utilizado también para la búsqueda parámetros concretos de los geles y membranas colágenas comercializados más utilizados actualmente (CarGel ${ }^{\circledR}$, CartiFill ${ }^{\circledR}$, Chondro-Gide $^{\circledR}$, ChondroFiller ${ }^{\circledR}$, De-Novo $\left.^{\circledR}, \mathrm{CaReS}^{\circledR}\right)$.

La selección final de trabajos revisados se ha realizado priorizando el nivel de evidencia, la metodología y la afinidad al objetivo de la revisión. Se ha revisado manualmente la bibliografía de cada uno de los trabajos seleccionados y muchos de sus resúmenes, para intentar no perder ningún artículo relevante. 
Se han encontrado dos revisiones sistemáticas sobre tratamiento de las lesiones osteocondrales del adulto en la librería Cochrane, una dedicada a lesiones en el astrágalo publicada en $2010^{(18)}$ y otra más reciente de 2016 que compara resultados de las diferentes técnicas para lesiones osteocondrales en la rodilla.

\section{Resultados}

Lo primero que llama la atención al analizar la bibliografía recopilada es la gran proliferación de trabajos relacionados con las lesiones del cartílago durante la última década, lo cual puede estar relacionado tanto con su prevalencia, con la controversia que aún existe en cuanto a la técnica de tratamiento más adecuada y con la proliferación de nuevas terapias.

Se han encontrado muy pocos ensayos de experimentación básica. La gran mayoría de los trabajos publicados en las revistas de nuestra especialidad son estudios clínicos, con series de casos y bajo nivel de evidencia. Hay pocos estudios prospectivos, aleatorizados y controlados, que comparen diferentes métodos de tratamiento, y menos aún con series de tamaño suficiente y seguimiento a medio o largo plazo, referidos concretamente al estudio de las nuevas terapias biológicas con geles y membranas colágenas sin cultivo celular.

\section{Evidencia general de terapias en lesiones osteocondrales de astrágalo}

Si empezamos por la CDSR, la base de datos de Revisiones Sistemáticas de la Biblioteca Cochrane, encontramos únicamente el trabajo publicado en 2010 por Loveday et al. ${ }^{(18)}$ referido anteriormente. Tiene como objetivo determinar los beneficios y efectos secundarios de las intervenciones para el tratamiento de las LOA en adultos. Basados en la existente controversia entre las diferentes técnicas de tratamiento, se plantean las hipótesis de tratar vs. no tratar, tratamiento quirúrgico vs. no quirúrgico, diferencias entre métodos no quirúrgicos y diferencias entre métodos quirúrgicos. Un único estudio ${ }^{(17)}$ cumplió los criterios de inclusión en cuanto a evidencia y metodología, y otro estaba aún incompleto. La conclusión de los autores es que no existe evidencia suficiente basada en ensayos clínicos aleatorizados y controlados para determinar cuál es el método de tratamiento más apropiado en LOA del adulto.

En una revisión sistemática publicada por Zengerink, del grupo de Van Dijk, en 2010(5), describen los resultados de 52 estudios (excluyen 202 por no cumplir criterios de inclusión) y solo uno aleatorizado. Aquí ya incluyen 4 trabajos sobre implante de condrocitos autólogos (ACl). Comentan que sobre todo el tamaño de la lesión puede ser un factor decisivo a la hora de elegir la técnica, pero que parece que sigue influyendo más la preferencia del cirujano. Concluyen en recomendar la EMO como técnica de elección para LOA primarias pero que, a la luz de la evidencia existente, no pueden obtener conclusiones definitivas. También destacan la mala calidad y diseño general de los estudios, y la necesidad de estudios clínicos aleatorizados y controlados (ECAC) con metodología y validación de resultados más homogéneas.

En una actualización sobre tratamiento quirúrgico de LOA de Murawski et al. de 2013(2) ya revisan la "segunda generación" del $\mathrm{ACl}$, implementada al cultivar los condrocitos sobre una membrana bioabsorbible de colágeno de tipo I/III de origen porcino, u otros tipos de matrices o geles basados en colágeno o ácido hialurónico (MACl). Hacen referencia a los trabajos de los grupos de Giannini, Magnan, Jones y Haleem, y destacan los beneficios que aportan estos "andamiajes" de colágeno, como evitar la necesidad de extraer e implantar un parche de periostio, permitir realizar todo el proceso mediante técnica artroscópica y mejor distribución celular en la membrana. Destacan los prometedores resultados publicados por los diferentes grupos, especialmente en lesiones más grandes, pero concluyen que no pueden establecerse indicaciones y recomendaciones definitivas. En el momento de la publicación, el método no está aceptado por la Food and Drug Administration (FDA) ni comercializado en los EE. UU. Dedican una sección a los "coadyuvantes biológicos" para la reparación del cartílago, revisando el concentrado de células mesenquimales aspirado de médula ósea y las infiltraciones de ácido hialurónico. Establecen unos grados de recomendación para las diferentes técnicas de tratamiento de LOA, destacando el bajo nivel de evidencia general (Tabla 1).

Recientemente, Pinski(8) ha realizado una revisión del nivel de evidencia y la calidad metodo- 


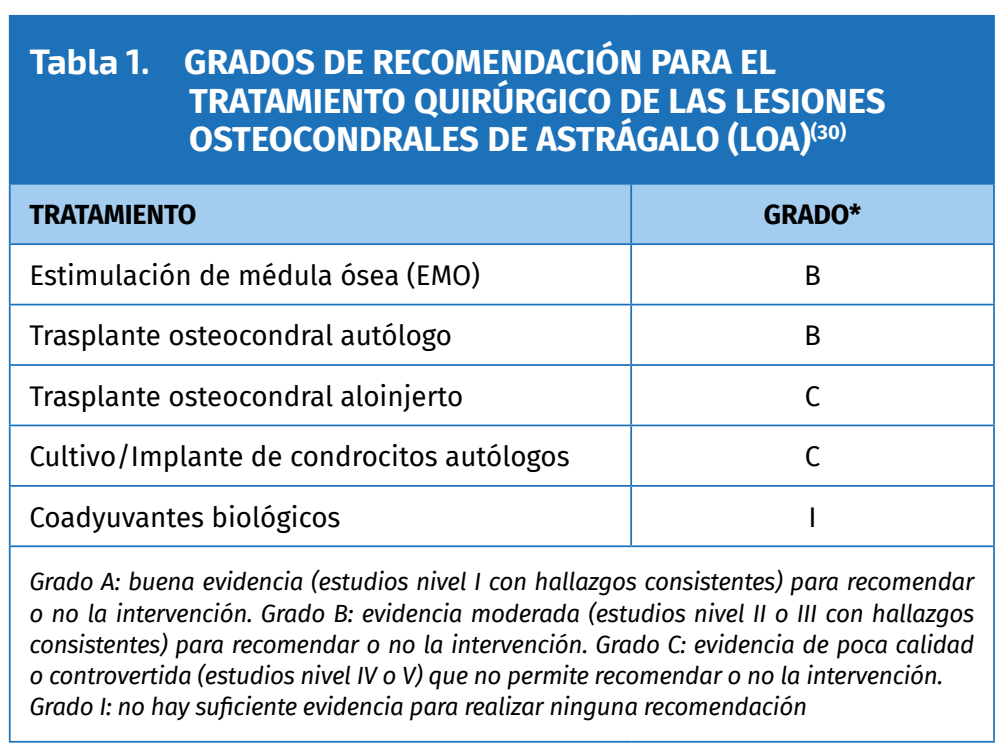

do, pero refieren el hallazgo de cartílago hialino en el $50 \%$ de los casos y mezcla de hialino-fibroso en el resto. Los resultados clínicos fueron buenos a los 2 años con una mejoría media de 35 puntos en la escala de Lysholm y 4 puntos de descenso del EVA en cuanto al dolor. El control por imagen en T2RM (resonancia magnética) demostró la existencia de un cartílago similar al hialino al año de seguimiento. Concluyen que $\mathrm{MACl}$ es superior a EMO aislada en cuanto a resultados clínicos a corto-medio plazo, pero no hay evidencia de que sea superior en cuanto a hallazgos en RM o histológicos. También destacan que no deberían compararse estas dos técnicas de forma general, ya que actualmente tienen indicaciones

lógica de los trabajos dedicados al tratamiento quirúrgico de las LOA. Tras evaluar los 83 estudios (2.425 procedimientos) más relevantes en función de sus criterios de inclusión, reporta que el 90\% obtuvo un nivel de evidencia IV y puntuó de media 53,6/100 en el CMS modificado (Coleman Methodology Score), por lo que alerta de que la gran mayoría de los estudios existentes sobre el tratamiento de las lesiones del cartílago en el tobillo tienen un nivel de evidencia muy bajo y una calidad metodológica pobre, y esto debe ser tenido en consideración a la hora de interpretar los resultados.

En 2015, Wylie et al. ${ }^{(19)}$ realizan una revisión sistemática planteándose el efecto de las matrices de colágeno en las técnicas reparadoras del cartílago. Seleccionan 17 artículos relevantes que cumplen los criterios de inclusión y en ellos identifican 11 técnicas diferentes basadas en matrices colágenas. Los más utilizados y referenciados en la bibliografía son Hyalograft $C^{\circledR}$ (Anika Therapeutics Inc, Bedford, MA, USA) y Chondro-Gide ${ }^{\circledR}$ (Genzyme Biosurgery, Kastrup, Denmark). Curiosamente, en la fecha de publicar el estudio, el primero ha sido retirado del mercado en Europa. A la hora de evaluar y comparar las diferentes técnicas y productos, de nuevo destacan la escasez de trabajos y su bajo nivel de evidencia. La comparación es dificil, ya que no siguen las mismas indicaciones (tamaño de la lesión, técnica, etc.). Hay pocos trabajos con estudio histológico y, cuando los hay, no están realizados en el mismo periodo postoperatorio, por lo que tampoco son comparables en este senti- diferentes. Y que no hay evidencia suficiente para poder comparar las diferentes matrices entre ellas. Incluso las más utilizadas y sobre las que existen más datos publicados tienen problemas de aprobación por la EMA.

\section{Uso de membranas asociado a trasplante de células derivadas de médula ósea (TCDMO)}

Varios autores han publicado el uso de membranas colágenas asociado a trasplante de concentrado de células mesenquimales pluripotenciales derivadas de médula ósea (TCDMO) con resultados prometedores ${ }^{(13,1,20,21)}$.

Buda et al., del grupo de Giannini, comparan dos grupos homogéneos de 40 pacientes, uno tratado mediante $\mathrm{MACl}$ y otro con la técnica de TCDMO que habían publicado previamente ${ }^{(21)}$, las dos técnicas de forma artroscópica. En ambos casos se utilizó una membrana de ácido hialurónico como soporte celular (HYAFF1-11, Anika Therapeutics, Bedford, MA). Los resultados clínicos a los 2 años fueron similares en ambos grupos y se confirmó mayor presencia de cartílago hialino en RM-T2 mapping en el grupo de TCDMO. Recomiendan la técnica de TCDMO dado que consigue resultados ligeramente superiores siendo más sencilla, requiere de un solo tiempo quirúrgico y con menor coste económico. No declaran conflicto de intereses. 


\section{Matrices de gel-colágeno}

\section{$\operatorname{CaReS}^{\circledR}$}

En 2011, Schneider publica un ensayo clínico en animales de experimentación comparando los resultados biohistológicos en cuanto a la reparación condral de 3 métodos de tratamiento. Realiza 3 lesiones en rodilla de 18 Gettinger minipigs adultos y las trata simultáneamente mediante un gel de colágeno de tipo I de forma aislada, sin aportación celular (CaReS ${ }^{\circledR}$, Arthro Kinetics, Esslingen); el mismo gel colágeno sembrado de condrocitos del mismo animal, previamente cultivados durante 2 semanas; y la tercera simplemente con microfracturas. Hay que resaltar que en los primeros 2 grupos no realiza microfracturas ni perforaciones en hueso subcondral. Realiza estudios de resistencia mecánica e inmunohistológicos del cartílago de reparación obtenido en los defectos a las 6, 12 y 52 semanas. Concluye que tanto el grupo tratado con gel únicamente como el enriquecido con cultivo de condrocitos presentaron una formación de cartílago de muy alta calidad, superior al grupo de microfracturas, y que las diferencias entre los 2 grupos de matriz colágena (con y sin aportación celular) fueron no significativas en los parámetros estudiados. Destaca la capacidad condrogénica de determinados geles o membranas, como el empleado en este estudio, y basándose en estudios previos advierte de que esta puede ser diferente en función de los componentes de dicho gel o membrana, y que se requieren más estudios en este campo. Aunque aún no existe evidencia y sigue siendo motivo de investigación, parece que la aportación celular en estos casos podría proceder de células mesenquimales del propio líquido sinovial(22) o del cartílago circundante. Esto puede suponer un importante avance en el tratamiento de las LOA, ya que la lesión del hueso subcondral, que puede incluso aumentar con la realización de microfracturas, también ha sido relacionada con peores resultados a medio plazo por disminución progresiva de la resistencia del cartílago suprayacente ${ }^{(23)}$.

\section{BST-CarGel ${ }^{\circledR}$}

Otra matriz-gel ampliamente difundida en nuestro entorno es el BST-CarGel ${ }^{\circledR}$ (Piramal Life Sciences, Laval, Quebec, Canada). Se trata de una matriz polimérica biosoluble, biocompatible y biodegradable, con $\mathrm{pH}$ y osmolaridad fisiológicas, con propiedades citomiméticas y alta adhesividad, gracias sobre todo a su contenido en chitosán, un polisacárido derivado del caparazón de los crustáceos. Por todas estas propiedades, es un biomaterial muy investigado y con múltiples aplicaciones biomédicas.

El BST-CarGel ${ }^{\circledR}$ es aplicado "en seco", en forma de coágulo adherente, tras ser mezclado con sangre autóloga en una proporción 1:3, y solidifica in situ en 10-15 min. Las propiedades del chitosán refuerzan el coágulo impidiendo su retracción y favorecen la adhesividad de la mezcla dentro de la lesión, evitando que se desprenda.

Desde 2006 se han publicado varios trabajos multicéntricos, aleatorizados y controlados, con series largas de lesiones en rodilla. En 2013 uno con nivel de evidencia I ha demostrado superioridad relativa en comparación con microfracturas en cuanto a calidad del cartílago neoformado, muy similar al hialino, pero no en cuanto a la clínica entre grupos, aunque ambos mejoraron significativamente. No evalúa el coste-beneficio.

Vilá y Rico et al.(24), en un trabajo multicéntrico dirigido en nuestro país en 2015, describen la técnica artroscópica en tobillo, siendo una de las ventajas de esta matriz, siempre que la lesión sea bien abordable. Recomiendan la técnica para lesiones de hasta $3 \mathrm{~cm}^{2}$ y describen su protocolo postoperatorio.

\section{Chondro-Gide ${ }^{\circledR}$}

Basándose en estudios previos y series publicadas sobre la técnica AMIC (autologous matrix-induced chondrogenesis) para grandes defectos condrales y osteocondrales en rodilla, Valderrabano et al. son los primeros en describir esta técnica en lesiones de astrágalo ${ }^{(25)}$. Incluyen una modificación realizando un autoinjerto previo de hueso esponjoso obtenido de cresta iliaca, con el doble objetivo de regenerar el hueso esclerótico o la lesión quística subcondral -como condición previa para un correcto sustrato anatómico donde asentar el cartílago neoformado- y el aporte de células mesenquimales de médula ósea. Esto les permite tratar lesiones grandes y con importantes defectos subcondrales que, según la evidencia existente, serían más susceptibles de autoinjerto 

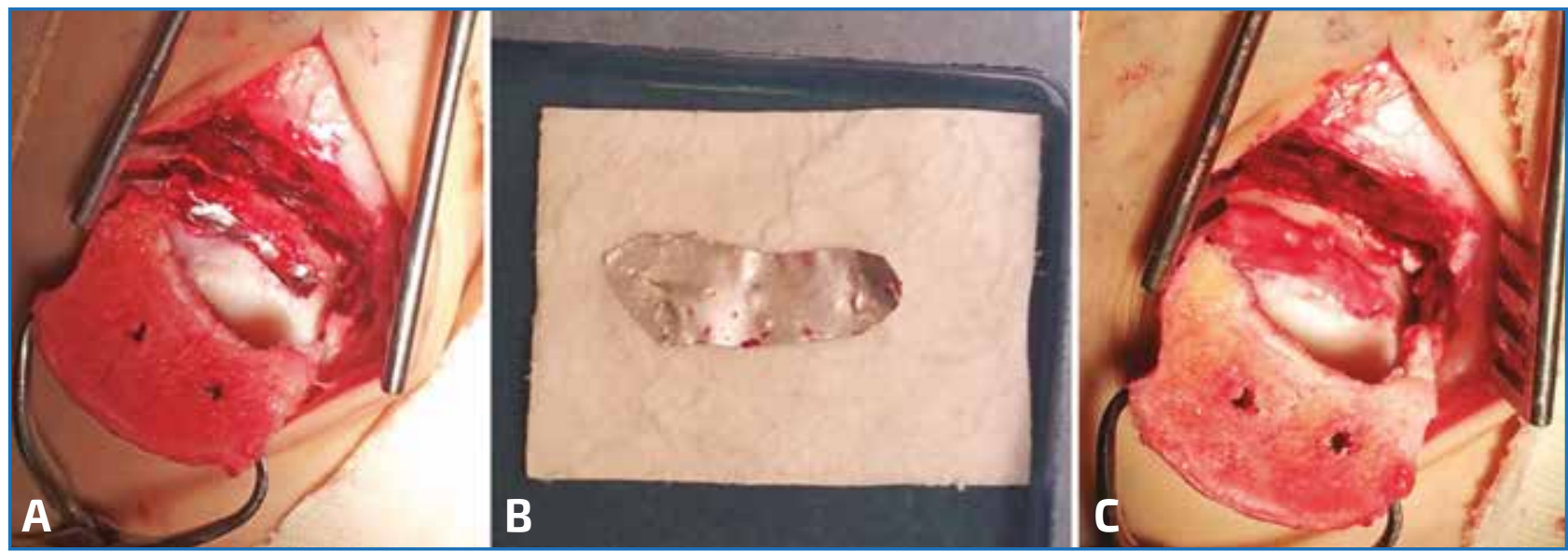

Figura 1. Rescate mediante AMIC (Chondro-Gide ${ }^{\circledR}$ ) de una recidiva de lesión osteocondral de astrágalo (LOA) medial tratada previamente con microfracturas. A: abordaje transmaleolar y toma de patrón de la lesión ya desbridada; B: tallado de la membrana; C: aspecto final tras sellado con fibrina.

osteocondral (OATS). Sobre este injerto implantan una membrana de colágeno I/III de origen porcino (Chondro-Gide ${ }^{\circledR}$, Geistlich Surgery, Wolhusen, Switzerland), que sellan con fibrina (Tissucol $\left.{ }^{\circledR}\right)$.

En su estudio prospectivo incluyen LOA de cualquier tamaño. Justifican el uso de artrotomías u osteotomías de maléolo lateral o medial, en la mayoría de los ocasos, por tratarse de lesiones grandes y para lograr buena exposición para el cruentado e injertado. Indican que puede realizarse de forma artroscópica en lesiones pequeñas y accesibles. En 2013 presentan buenos resultados a 2 años ${ }^{(25)}$ en una serie de 26 pacientes (17-55 años), con mejoría de AOFAS (American Orthopedic Foot and Ankle Score) hasta 89 puntos y EVA 1,6 postoperatorios. El 45\% de los pacientes recuperó su nivel de actividad deportiva prelesional. También describen importante mejoría radiológica, con señal en RM "casi normal" y similar al cartílago sano circundante en el 65\%, y MOCART (magnetic resonance observation of cartilage repair tissue) medio de 62/100, equiparable a lo reportado en series recientes con técnica $\mathrm{MACl}$. No refieren ninguna complicación y hacen referencia a un estudio económico que demuestra el coste-efectividad de esta membrana (Figura 1).

\section{CartiFill ${ }^{\circledR}$}

Otra matriz-gel usada en nuestro medio es el CartiFill ${ }^{\circledR}$ (Joint Biomaterials SAS, Mestre, Italy). Se

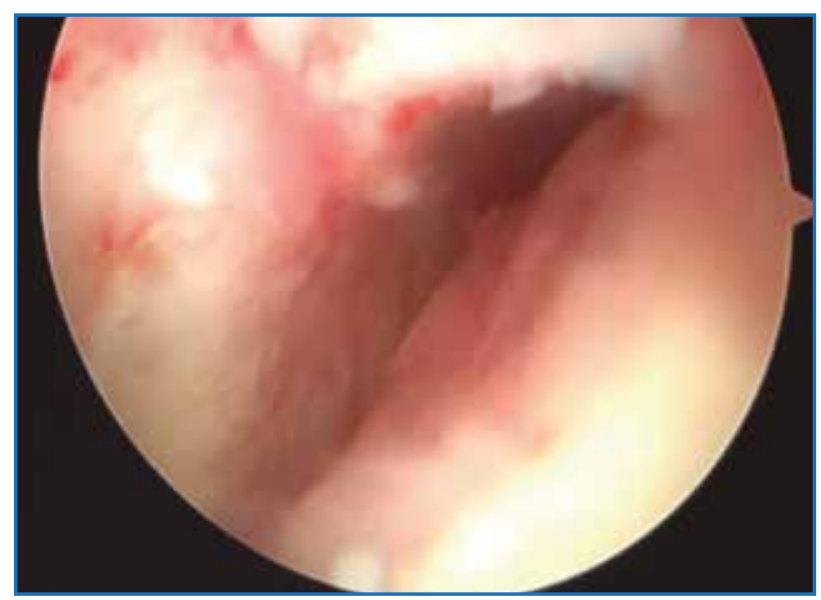

Figura 2. Tratamiento artroscópico mediante AGIC (CartiFill ${ }^{\circledR}$ ).

trata de atelocolágeno aislado de dermis porcina enriquecida con nutrientes esenciales para la anidación y el cultivo de células mesenquimales. Se inyecta en seco a través del portal artroscópico, simultáneamente con fibrina (Tissucol ${ }^{\circledR}$ ) en proporción 1:1, mediante una cánula en $Y$, polimerizando en 6-8 minutos. Pueden añadirse coadyuvantes biológicos como PRP. Usuelli et al. ${ }^{(26)}$ publican una serie de 9 pacientes con LOA pequeñas $\left(2,1 \pm 0,9 \mathrm{~cm}^{2}\right)$ intervenidos mediante esta técnica que denominan ACIC (autologous collagen-induced chondrogenesis) con un año de seguimiento. Destacan los buenos resultados clínicos en cuanto a función y dolor, la seguridad del 
implante, la sencillez de la técnica (un tiempo y artroscópica) y el bajo coste en comparación con otros tratamientos. Las imágenes RM mostraron un MOCART de 51,7 $\pm 16,6$, algo inferior a otras series, que ellos relacionan con la existencia aún de cartílago inmaduro en formación, dado el escaso tiempo de evolución. No encontraron correlación del MOCART con la edad, índice de masa corporal, tamaño de la lesión, ni con la mejoría clínica en escala AOFAS o EVA. Reconocen las limitaciones de la serie pequeña, el poco tiempo de evolución, no grupo control y los escasos datos existentes en cuanto a la calidad del cartílago de reparación obtenido (Figura 2).

\section{Discusión}

La EMO subcondral sigue siendo la primera opción y el método más utilizado para el tratamiento de LOA pequeñas ${ }^{(4,5,11-13,27)}$. En lesiones más grandes, parece que la evidencia existente recomienda técnicas de implante de condrocitos ( $\mathrm{ACl} / \mathrm{MACl}$ ) o autoinjerto osteocondral (OATS). El aloinjerto se reserva para grandes defectos o rescates.

Aunque sigue siendo tema de controversia, parece que la edad (> 45 años) y el tamaño de la lesión (> $15 \mathrm{~mm}$ ) pueden ser factores limitantes en cuanto al pronóstico y la elección del método de tratamiento ${ }^{(12,23)}$. Sin embargo, una de las mayores series y con resultados a más largo plazo (12 años), publicada por Van Bergen ${ }^{(27)}$ concluía recomendando la EMO por sus buenos resultados y mantenidos en el tiempo hasta en un $78 \%$ de los pacientes, independientemente de la edad y el tamaño de la lesión.

Otros autores han observado deterioro clínico y radiológico a partir de los 5 años. Este resultado se relaciona con la menor resistencia mecánica del fibrocartílago de reparación obtenido, por lo que técnicas alternativas persiguen la formación un cartílago hialino rico en colágeno de tipo II, con mayor resistencia a largo plazo, a pesar de que esta relación aún no ha podido ser confirmada.

Con este objetivo, se investigó en cultivo e implante de condrocitos autólogos ( $\mathrm{ACl}$ ) desde finales de los noventa y, para implementar y simplificar la técnica, se diseñaron matrices de colágeno o hialurónico sobre los que "sembrar" los condrocitos cultivados (MACl). En los últimos años, gracias a la evolución de estos geles o membranas y a progresos en el campo de la biología celular y molecular aplicada, parece que se pueden obtener similares resultados clínicos y en calidad y cantidad del cartílago neoformado, pero de forma más sencilla y económica, en un solo tiempo quirúrgico, aportando a estos "andamiajes" de colágeno concentrados de células mesenquimales obtenidas de médula ósea (TCDMO) capaces de diferenciarse en condrocitos $^{(21,28)}, \mathrm{o}$, incluso, sin ningún aporte celular "externo", obteniendo las células progenitoras indiferenciadas de la médula ósea subcondral de la lesión o desde el propio fluido sinovial o cartílago circundante ${ }^{(22)}$. En función de estos últimos trabajos, cuyos mecanismos por medio de moduladores de diferenciación celular aún necesitan ser establecidos, se obtendrían resultados similares, sin necesidad de perforar o debilitar el hueso subcondral, aumentando el poder condrogénico y modulador de las matrices colágenas.

Estas técnicas de estimulación de condrogénesis autóloga mediante membranas (AMIC) o geles (ACIC), asociadas a perforaciones (micro- o nanofracturas), podrían denominarse de forma general "EMO aumentada"(10,29). La matriz de base colágena tiene la doble función de andamiaje sobre el que "anidan" las células mesenquimales $\mathrm{y}$, por otro, "contener" y estabilizar el hematoma en la lesión, logrando así un ambiente biológicamente propicio para la proliferación y diferenciación celular ${ }^{(2,4,16,19,24,28)}$.

En torno a esta tendencia cuyos resultados clínicos parecen prometedores, se ha desarrollado una industria que actualmente fabrica y promociona muchos y diferentes productos. A pesar de que pueden variar en su composición y características, aún no se han podido demostrar diferencias significativas en cuanto a resultados clínicos entre ellos ${ }^{(8,10,19)}$.

Algunas matrices han tenido problemas de aprobación y la mayoría no están aún aceptadas por la FDA. Todas adolecen de evidencia suficiente para ser recomendados como tratamiento de elección $n^{(8,19)}$.

Está demostrado que, en líneas generales, se publican más estudios con resultados positivos y que estos tienden a ser publicados en revistas de mayor índice de impacto. También que las nuevas técnicas se publican más si sus resultados son buenos. Todo ello podría llevar a sobreestimar los efectos de estas nuevas terapias ${ }^{(19)}$. 
La mayor resistencia mecánica y estabilidad que los geles o membranas imprimen al coágulo, así como un mejor aislamiento del medio intraarticular que evite migración celular o de factores inflamatorios no deseados al interior de la lesión o hueso subcondral, podría interferir con el proceso reparativo y en algunos trabajos se ha relacionado este hecho con la principal causa de dolor en las LOA, dado que el cartílago en sí mismo es un tejido anervado(25). Sin embargo, la ventaja de los geles respecto a las membranas puede ser su facilidad técnica para implantarlos por vía artroscópica ${ }^{(24,26)}$.

Una presentación a medio camino podrían ser las matrices de gel sólido prensado (CaRes ${ }^{\circledR}$, ChondroFiller ${ }^{\circledR}$ ), con diferentes tamaños para ser adaptado a la lesión de tipo press-fit. A pesar de la aparente estabilidad, algunos autores recomiendan reforzar la fijación con fibrina.

Aunque la mayoría de los trabajos parecen demostrar resultados beneficiosos con el uso de matrices colágenas, superiores a microfracturas y con muy escasas complicaciones, no existe aún evidencia suficiente al respecto. Las series son muy heterogéneas y las indicaciones muy irregulares, por lo que resulta complicado comparar los resultados. Además, la mayoría de las publicaciones son series de casos y con poco seguimiento. Hay una importante falta de estudios con metodología adecuada y tiempo de evolución suficiente para poder sacar conclusiones al respecto ${ }^{(8,77)}$.

Deberían compararse las diferentes matrices existentes entre ellas y también con otras técnicas alternativas para lesiones más grandes, como el implante de condrocitos autólogos (MACl) o el autoinjerto osteocondral (OATS), y no únicamente con las técnicas de EMO. Para el correcto diseño de estos estudios, deberían homogeneizarse previamente las indicaciones y los métodos de valoración de resultados, tanto clínicos como radiológicos e histológicos.

\section{Conclusiones}

- El uso de matrices de colágeno como tratamiento de LOA en el adulto ha despertado el interés de científicos y cirujanos ortopédicos en los últimos años y su utilización ha proliferado de forma heterogénea.

- A pesar de la gran cantidad de estudios recientemente publicados, no hay evidencia sufi- ciente para poder recomendar este método de tratamiento en LOA grandes o pequeñas.

- Aun así, los trabajos publicados muestran resultados satisfactorios a corto y medio plazo, sobre todo para LOA pequeñas, asociando técnicas de EMO o aporte de células mesenquimales (TCDMO). En lesiones más grandes, a pesar de que estos resultados no son superiores a técnicas de implante de condrocitos autólogos (MACl) o autoinjerto osteocondral (OATS), dada su mayor simplicidad y buena relación coste-efectividad, tanto AGIC (autologous gel-induced condrogenesis) como AMIC (autologous matrix-induced condrogenesis) pueden suponer una buena alternativa.

- Faltan mejores estudios multicéntricos, prospectivos, aleatorizados y controlados, a medio-largo plazo, para poder definir los usos e indicaciones de estas nuevas terapias.

\section{Bibliografía}

1. Morales ], García E, Asunción J, Poggio D. [Medium-term results of arthroscopic debridement in osteochondral talar lesions]. Acta Ortop Mex. 27(5):319-23.

2. Murawski CD, Kennedy JG. Operative treatment of osteochondral lesions of the talus. J Bone Jt Surg Am. 2013;95(11):1045-54.

3. Henkelmann R, Schmal H, Pilz IH, Salzmann GM, DoviAkue D, Südkamp NP. Prospective clinical trial of patients who underwent ankle arthroscopy with articular diseases to match clinical and radiological scores with intra-articular cytokines. Int Orthop. 2015;39(8):1631-7.

4. Gianakos AL, Yasui Y, Hannon CP, Kennedy JG. Current management of talar osteochondral lesions. World J Orthop. 2017;8(1):12.

5. Zengerink M, Struijs PAA, Tol JL, van Dijk CN. Treatment of osteochondral lesions of the talus: a systematic review. Knee Surg Sports Traumatol Arthrosc. 2010;18(2):238-46.

6. Mellado Romero M, Bravo Giménez B, Martín López CM, Cano Egea JM, Vilá y Rico J, Resines Erasun C. Tratamiento artroscópico de las lesiones osteocondrales de astrágalo: estudio de 73 casos. Rev Esp Cir Ortop Traumatol. 2013;57(3):224-30.

7. Loveday D, Clifton R, Robinson A. Interventions for treating osteochondral defects of the talus in adults. Cochrane Database Syst Rev. 2009;(4).

8. Pinski JM, Boakye LA, Murawski CD, Hannon CP, Ross KA, Kennedy JG. Low Level of Evidence and Methodologic 
Quality of Clinical Outcome Studies on Cartilage Repair of the Ankle. Arthroscopy. 2016;32(1):214-22.e1.

9. Vannini F, Costa GG, Caravelli S, Pagliazzi G, Mosca M. Treatment of osteochondral lesions of the talus in athletes: what is the evidence? Joints. 2016;4(2):111-20.

10. Yasui Y, Wollstein A, Murawski CD, Kennedy JG. Operative Treatment for Osteochondral Lesions of the Talus: Biologics and Scaffold-Based Therapy. Cartilage. 2016;8(1):1947603516644298.

11. Gobbi A, Francisco RA, Lubowitz JH, Allegra F, Canata G. Osteochondral lesions of the talus: randomized controlled trial comparing chondroplasty, microfracture, and osteochondral autograft transplantation. Arthroscopy. 2006;22(10):1085-92.

12. Ramponi L, Yasui Y, Murawski CD, Ferkel RD, DiGiovanni CW, Kerkhoffs GM, et al. Lesion Size Is a Predictor of Clinical Outcomes After Bone Marrow Stimulation for Osteochondral Lesions of the Talus: A Systematic Review. Am J Sports Med. 2016:363546516668292.

13. Savage-Elliott I, Ross KA, Smyth NA, Murawski CD, Kennedy JG. Osteochondral lesions of the talus: a current concepts review and evidence-based treatment paradigm. Foot Ankle Spec. 2014;7(5):414-22.

14. Buda R, Vannini F, Castagnini F, Cavallo M, Ruffilli A, Ramponi $L$, et al. Regenerative treatment in osteochondral lesions of the talus: autologous chondrocyte implantation versus one-step bone marrow derived cells transplantation. Int Orthop. 2015;39(5):893-900.

15. Giannini S, Battaglia M, Buda R, Cavallo M, Ruffilli A, Vannini F. Surgical Treatment of Osteochondral Lesions of the Talus by Open-Field Autologus Chondrocyte Implantation. Am J Sports Med. 2009;37(Supplement 1):112S-118S.

16. Buda R, Vannini F, Castagnini F, Cavallo M, Ruffilli A, Ramponi L, et al. Regenerative treatment in osteochondral lesions of the talus: autologous chondrocyte implantation versus one-step bone marrow derived cells transplantation. Int Orthop. 2015;39(5):893-900.

17. Doral MN, Bilge O, Batmaz G, Donmez G, Turhan E, Demirel $M$, et al. Treatment of osteochondral lesions of the talus with microfracture technique and postoperative hyaluronan injection. Knee Surg Sports Traumatol Arthrosc. 2012;20(7):1398-403.

18. Loveday D, Clifton R, Robinson A. Interventions for treating osteochondral defects of the talus in adults. Cochrane Database Syst Rev. 2010;(8):CD008104.

19. Wylie JD, Hartley MK, Kapron AL, Aoki SK, Maak TG. What Is the Effect of Matrices on Cartilage Repair? A Systematic Review. Clin Orthop Relat Res. 2015;473(5):1673-82.

20. Looze CA, Capo J, Ryan MK, Begly JP, Chapman C, Swanson D, et al. Evaluation and Management of Osteo- chondral Lesions of the Talus. Cartilage. 2017;8(1): 19-30.

21. Buda R, Castagnini F, Cavallo M, Ramponi L, Vannini F, Giannini S. One-step bone marrow-derived cells transplantation and joint debridement for osteochondral lesions of the talus in ankle osteoarthritis: clinical and radiological outcomes at 36months. Arch Orthop Trauma Surg. 2016;136(1):107-16.

22. Kim YS, Lee HJ, Yeo JE, Kim Y IL, Choi YJ, Koh YG. Isolation and characterization of human mesenchymal stem cells derived from synovial fluid in patients with osteochondral lesion of the talus. Am J Sport Med. 2015;43(2):399-406.

23. Yoshimura I, Kanazawa K, Takeyama A, Angthong C, Ida T, Hagio T, et al. Arthroscopic bone marrow stimulation techniques for osteochondral lesions of the talus: prognostic factors for small lesions. Am J Sports Med. 2013;41(3):528-34.

24. Vilá y Rico J, Dalmau A, Chaqués FJ, Asunción J. Treatment of Osteochondral Lesions of the Talus With Bone Marrow Stimulation and Chitosan-Glycerol Phosphate/Blood Implants (BST-CarGel). Arthrosc Tech. 2015;4(6):e663-e667.

25. Valderrabano V, Miska M, Leumann A, Wiewiorski M. Reconstruction of osteochondral lesions of the talus with autologous spongiosa grafts and autologous matrix-induced chondrogenesis. Am J Sports Med. 2013;41(3):519-27.

26. Usuelli FG, Grassi M, Manzi L, Guarrella V, Boga M, De Girolamo $L$. Treatment of osteochondral lesions of the talus with autologous collagen-induced chondrogenesis: clinical and magnetic resonance evaluation at one-year follow-up. Joints. 2016;4(2):80-6.

27. Van Bergen CJA, Kox LS, Maas M, Sierevelt IN, Kerkhoffs GMMJ, van Dijk CN. Arthroscopic treatment of osteochondral defects of the talus: outcomes at eight to twenty years of follow-up. J Bone Joint Surg Am. 2013;95(6):519-25.

28. Chahla J, Cinque ME, Shon JM, Liechti DJ, Matheny LM, LaPrade RF, Clanton TO. Bone marrow aspirate concentrate for the treatment of osteochondral lesions of the talus: a systematic review of outcomes. J Exp Orthop. 2016:3(1):33.

29. Khazzam M. Augmented microfracture: is this the Holy Grail that we have been searching for in the treatment of cartilage injuries?: commentary on an article by William D. Stanish, MD, et al.: "Novel scaffold-based BST-CarGel treatment results in superior cartilage repair compared with microfracture in a randomized controlled trial". J Bone Joint Surg Am. 2013;95(18):e137.

30. Murawski CD, Kennedy JG. Operative treatment of osteochondral lesions of the talus. J Bone Jt Surg Am. 2013;95(11):1045-54. 